\title{
Seyfert 2 galaxies in the GeV band: jets and starburst
}

\author{
J.-P. Lenain, C. Ricci, M. Türler, D. Dorner, R. Walter \\ ISDC Data Centre for Astrophysics, Observatoire de Genève, Université de Genève, Chemin \\ d'Ecogia 16, 1290 Versoix, Switzerland \\ E-mail: jean-philippe.lenain@unige.ch
}

\begin{abstract}
The Fermi/LAT collaboration recently reported the detection of starburt galaxies in the high energy $\gamma$-ray domain, as well as radio-loud narrow-line Seyfert 1 objects, which were previously unusual extragalactic suspects for high energy emission. Motivated by the presence of sources close to the location of composite starburst/Seyfert 2 galaxies in the first year Fermi/LAT catalogue, we studied high energy $\gamma$-ray emission from such objects, aiming at disentangling the emission of starburst and Seyfert activity. We analysed 1.6 years of Fermi/LAT data from NGC 1068 and NGC 4945, which count among the brightest Seyfert 2 galaxies. We found an excess of high energy $\gamma$-rays of $8.3 \sigma$ and $9.2 \sigma$ for 1FGL J0242.7+0007 and 1FGL J1305.4-4928, which are found to be consistent with the position of the Seyfert 2 galaxies NGC 1068 and NGC 4945, respectively. For both sources, we detect no significant variability nor any indication of a curvature of the spectrum. While the high energy flux of NGC 4945 is consistent with starburst activity, that of NGC 1068 is an order of magnitude above expectations, suggesting dominant emission from the active nucleus. We show that a leptonic scenario can account for the multi-wavelength spectral energy distribution of NGC 1068. High energy $\gamma$-ray emission is thus revealed for the first time in a Seyfert 2 galaxy. If this result is confirmed in other objects, new perspectives would be opened up into the $\mathrm{GeV}$ band, with the discovery of a new class of high energy $\gamma$-ray emitters.
\end{abstract}

25th Texas Symposium on Relativistic Astrophysics - TEXAS 2010

December 06-10, 2010

Heidelberg, Germany 


\section{Introduction}

The Fermi/LAT collaboration recently reported the discovery of four radio-loud narrow-line Seyfert 1 galaxies [1,2]. Previously undetected at high energies, these objects thus constitute a new class of high energy emitters. Extending this idea to Seyfert 2 galaxies, we searched for high energy emission from two active galactic nuclei (AGN) among the closest and brightest in the X-ray sky, NGC 1068 and NGC 4945.

NGC 1068 is an archetypal Seyfert 2 galaxy, located at $z=0.003786$, i.e. $14.4 \mathrm{Mpc}$ away, harboring a hidden Seyfert 1 core. Antonucci \& Miller proposed the AGN unification based on studies of this source [3]. Both AGN and starburst activities are present in the central region of NGC 1068 [4, 5], the latter dominating the infrared emission of the broadband spectral energy distribution (SED) [6].

The presence of a Fermi/LAT source, 1FGL J0242.7+0007, in the region of NGC 1068 in the 11-months Fermi/LAT catalogue (1FGL) [7], without any proposed counterpart in radio nor in $\gamma$ rays, motivated us to analyse 1.6 years of data from Fermi/LAT to better assess the origin of this $\gamma$-ray emission.

NGC 4945, another Seyfert 2 galaxy at $z=0.001908$, also exhibits starburst activity within its core. Its emission was detected up to soft $\gamma$-rays, as observed with INTEGRAL/SPI, and was found to be Compton thick [8]. This source was reported as a $\gamma$-ray emitter in the 1FGL catalogue, although the authors did not conclude on the starburst or AGN origin of the emission.

\section{Data analysis}

We analysed $\sim 1.6$ yr of Fermi/LAT data, spanning from August 4, 2008 to March 15, 2010, from a region of interest of $10^{\circ}$ in radius around NGC 1068, using the publicly available Science Tools $^{1}$, and we followed the unbinned likelihood analysis scheme [9].

Assuming a power-law shape for the source spectrum, the Test Statistic (TS) [10] of the likelihood analysis is 68.6 , corresponding approximately to a $8.3 \sigma$ source detection in the $100 \mathrm{MeV}-$ $100 \mathrm{GeV}$ range. The best-fit location of the source is $\alpha_{J 2000}=2^{\mathrm{h}} 42^{\mathrm{m}} 46^{\mathrm{s}}, \delta_{J 2000}=0^{\circ} 2^{\prime} 14^{\prime \prime}$ with an error circle radius of $6.1^{\prime}$ ( $68 \%$ confidence level, CL), and is fully compatible with the position reported in the 1FGL catalogue. Given the angular distance between the Fermi source 1FGL J0242.7+0007 and NGC 1068, and the optical extension of $6.5^{\prime}$ for the host galaxy, we propose that this Fermi/LAT source is actually associated with the Seyfert 2 galaxy NGC 1068.

All the sources reported in the Fermi/LAT 11-months catalogue [7] within a radius of $15^{\circ}$ around NGC 1068 were included in the likelihood analysis, and modelled with power-law spectra. For NGC 1068, we obtain $F_{100 \mathrm{MeV}-100 \mathrm{GeV}}=(8.6 \pm 2.3) \times 10^{-12} \mathrm{erg} \mathrm{cm}^{-2} \mathrm{~s}^{-1}$ and $\Gamma=2.31 \pm 0.13$. We also tried to fit the data with a broken power-law or a log parabola, but this did not improve the likelihood. No significant variability was found in the data.

We followed the same procedure for the analysis of Fermi/LAT data in the region of NGC 4945, for the source 1FGL J1305.4-4928. The likelihood analysis on NGC 4945 results in a TS of 85.3 in the $100 \mathrm{MeV}-100 \mathrm{GeV}$ band, equivalent to a $9.2 \sigma$ detection. The nominal position of the $\gamma$-ray emission, located at $\alpha_{J 2000}=13^{\mathrm{h}} 05^{\mathrm{m}} 33^{\mathrm{s}}, \delta_{J 2000}=-49^{\circ} 26^{\prime} 44^{\prime \prime}$ with an error circle radius of $3.2^{\prime}$

\footnotetext{
${ }^{1}$ http://fermi.gsfc.nasa.gov/ssc/data/analysis/software/
} 


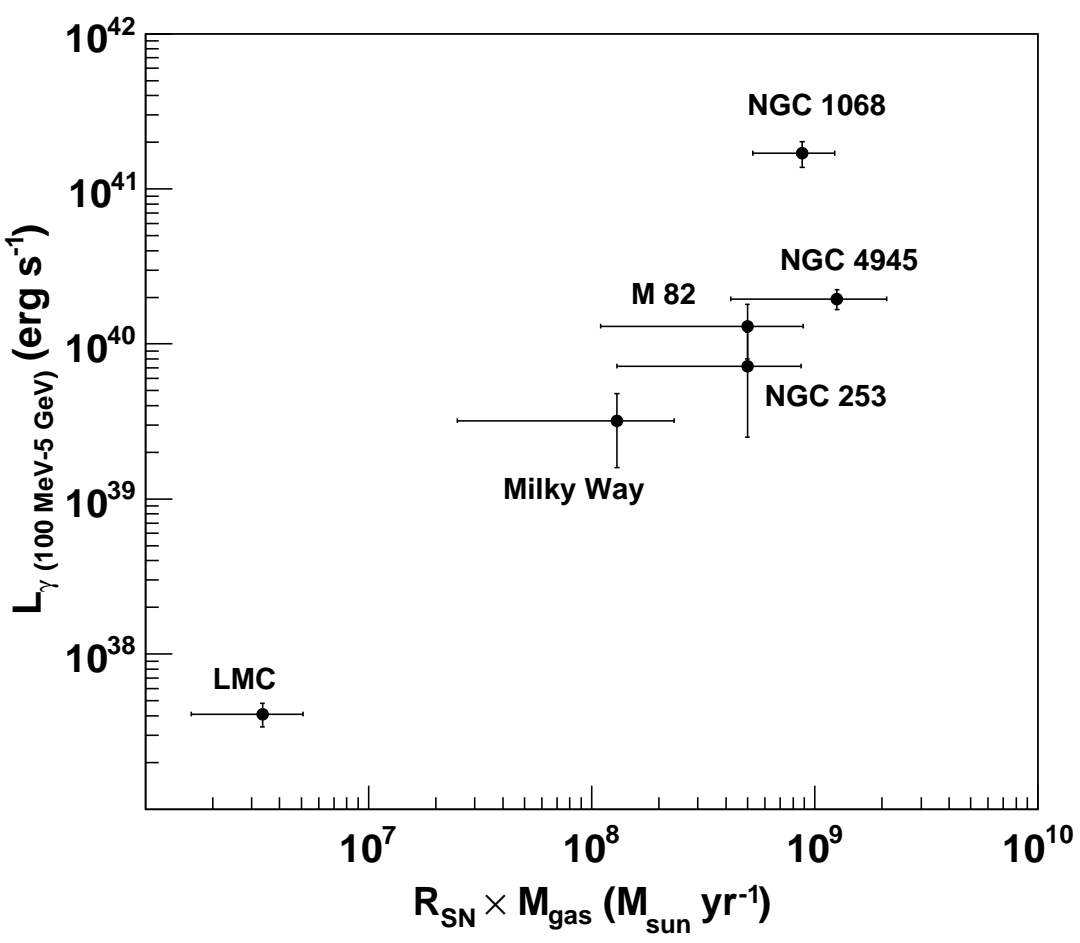

Figure 1: Relationship between SN rate, total gas mass and $\gamma$-ray luminosity of NGC 1068, NGC 4945, NGC 253, M 82, the LMC and the Milky Way.

(68\% CL), is only $1.6^{\prime}$ away from the position of NGC 4945. Assuming a power-law shape on the source energy spectrum, a photon index of $\Gamma=2.31 \pm 0.10$ and a flux of $F_{100 \mathrm{MeV}-100 \mathrm{GeV}}=(1.6 \pm$ $0.3) \times 10^{-11} \mathrm{erg} \mathrm{cm}^{-2} \mathrm{~s}^{-1}$ are found. As for NGC 1068, the use of a broken power-law or a log parabola did not improve the likelihood, and no significant variability was found in the data, which are statistically consistent with a constant for both sources.

The ISGRI data were reduced using the INTEGRAL Offline Scientific Analysis software ${ }^{2}$ version 9.0. We have also checked the Swift/BAT [11] spectra of the two sources. The 18 months Swift/BAT spectra have been extracted from the BAT archive [12] served by the HEAVENS source results archive (Walter et al., in prep.).

\section{Discussion}

As a first test to know whether the high energy emission of these two objects orginates from the starburst or the AGN component, a significant detection of variability would rule out the starburst hypothesis. However, due to the lack of statistics, no conclusions can be drawn about the variability of NGC 1068 or NGC 4945 from their light curves.

We also compare their $\gamma$-ray luminosity with those of the famous starburst galaxies NGC 253 and M 82, both detected at high energies with Fermi/LAT [13] and at very high energies with

\footnotetext{
${ }^{2} \mathrm{http} / / / \mathrm{www}$. isdc.unige.ch/integral/
} 


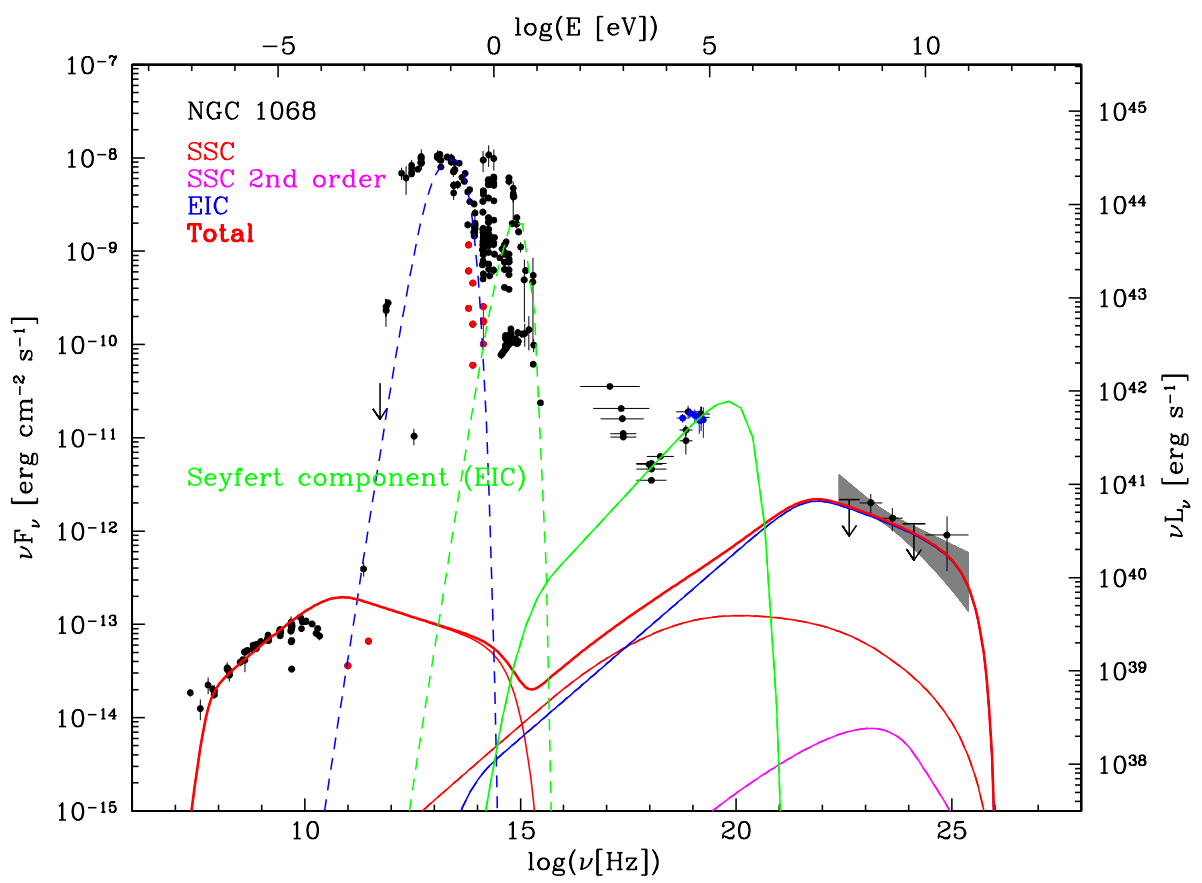

Figure 2: Spectral energy distribution of NGC 1068, including the Fermi/LAT spectrum. The black and red points are archival data from the NED, the red ones denote data taken from the central region of NGC 1068. For clarity, we only show the INTEGRAL IBIS/ISGRI data in blue in the hard X-rays. The EIC model for the outflow is shown in blue, and the corresponding SSC emission is shown in thin red and magenta lines for first and second order components, respectively. The thick red line shows the sum of the different emission components from the large outflow. The EIC component from the accretion disc is shown in green.

H.E.S.S. [14] and VERITAS [15], respectively. Both have a luminosity in the $100 \mathrm{MeV}-5 \mathrm{GeV}$ band of the order of $\approx 10^{40} \mathrm{erg} \mathrm{s}^{-1}$, as detected with Fermi [13]. Computing the luminosities of NGC 1068 and NGC 4945 in the same energy band, for comparison, we obtain $1.7 \times 10^{41} \mathrm{erg} \mathrm{s}^{-1}$ and $2.0 \times 10^{40} \mathrm{erg} \mathrm{s}^{-1}$, respectively. High energy emission from starburst galaxies is thought to originate from the interactions of cosmic rays produced by supernovæ with the ambient interstellar medium, and the $\gamma$-ray luminosity $L_{\gamma}$ should then scale with the product of the supernova rate $R_{\mathrm{SN}}$ and the total gas mass $M_{\text {gas }}$. We thus compare $R_{\mathrm{SN}}, M_{\text {gas }}$ and $L_{\gamma}$ of NGC 1068 and NGC 4945 to the ones of NGC 253, M 82, the Large Magellanic Cloud (LMC) and the Milky Way (see Figure 1), as well as their infrared and radio luminosities (see [16] for more details). These objects are the only extragalactic sources which are not AGN known to emit high energy $\gamma$-rays. The $\gamma$-ray luminosity and the SN rate of NGC 4945 are fully consistent with those of NGC 253 and M 82, hence even though this object is a composite starburst/AGN, its high energy $\gamma$-ray emission could be explained only in terms of starburst activity.

However, in the case of NGC $1068, R_{\mathrm{SN}}$ is comparable to those of M 82 and NGC 253, but its radio and $\gamma$-ray luminosities are higher by a factor $\sim 10$. This would suggest that its high energy $\gamma$ ray emission is more likely dominated by the central AGN activity. This is also strengthened by the fact that radio maps of NGC 1068 clearly show a structured jet, on parsec- and kiloparsec-scales, shaped by the outflow from the central AGN $[17,18]$. On the contrary, the radio morphology of 


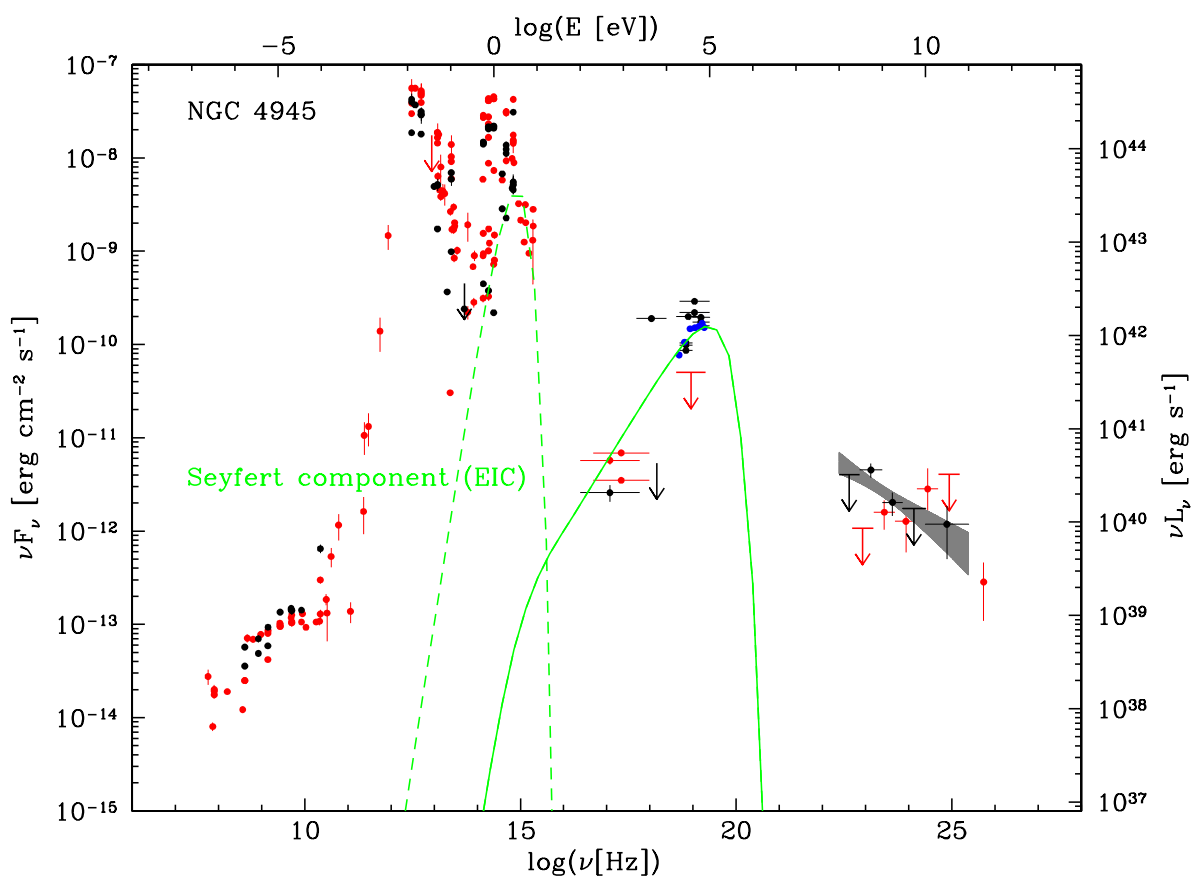

Figure 3: Spectral energy distribution of NGC 4945, including the Fermi/LAT spectrum (black points). For clarity, we only show the INTEGRAL IBIS/ISGRI data in blue in the hard X-rays. The model for the EIC component from the accretion disc is shown in green. We show in red the data of NGC 253 as taken from the NED, with its Fermi/LAT spectrum [13] as well as the H.E.S.S. flux measurement [14], for comparison. The luminosity axis on the right is given for NGC 4945.

NGC 4945 shows an extended emission consistent with the optical morphology tracing the edge-on galaxy, consistent with a starburst emission.

Assuming that the $\gamma$-ray emission of NGC 1068 is due to the central AGN, we apply an emission model developed for AGN which jet is misaligned to the line of sight [19]. A large, mildlyrelativistic zone of the wind-like outflow, at a few tenth of parsecs from the core, could emit highenergy $\gamma$-rays through external inverse Compton process (EIC) [20]. At such distances, the infrared photon energy density is still high enough to ensure a significant EIC emission while being not too important to prevent high optical opacity from pair production. In accordance with the data, no significant short-term variability is expected from such a large emitting zone.

In the model, a compact, dense plasma radiates through synchrotron self-Compton (SSC) and EIC processes. Second order SSC emission is also accounted for. Figure 2 shows the results of our model, with synchrotron emission responsible for the radio emission, while the Fermi/LAT data are interpreted as EIC emission with the infrared emission providing the seed photons, from a multitemperature blackbody. We also show the contribution from the SSC process, which is negligible compared to the EIC emission at the highest energies (see [16] for more details). The hard X-ray spectrum is due to EIC radiation from another relativistic population of leptons, within hot plasma located in the vicinity of the accretion disc. It should be noted that imaging atmospheric Čerenkov telescopes have the ability to strongly constrain the highest energy part of the particle energy distribution, and hence provide insights on the acceleration processes at work in this object. If the 
leptonic population of particles extend at higher energies, a significant signal should be detectable from NGC 1068 with H.E.S.S., VERITAS, MAGIC or the future CTA (Čerenkov Telescope Array) observatory [21].

Figure 3 shows the SED of NGC 4945 including archival data, as well as the Fermi/LAT and INTEGRAL spectra. We also show for comparison the SED of the starburst galaxy NGC 253. The two sources clearly have very similar broadband SEDs, strengthening the idea that the high energy $\gamma$-ray emission from NGC 4945 is of starburst origin. NGC 253 being detected at very high energies with H.E.S.S. [14], NGC 4945 is thus expected to be also detectable in this energy range.

\section{Conclusion}

High energy $\gamma$-ray emission is revealed and associated to the starburst/Seyfert 2 objects NGC 1068 and NGC 4945, using Fermi/LAT data. Both source spectra are consistent with a powerlaw, and no variability is found in the data. Compared to M 82 and NGC 253, which high-energy emission is dominated by starburst activity, we find a too high $\gamma$-ray luminosity of NGC 1068 to be explained only by starburst activity. We thus propose a leptonic scenario to interpret the high energy emission in terms of external inverse Compton process from an outflowing relativistic wind launched by the central AGN.

If high energy $\gamma$-ray emission due to AGN activity is confirmed in other Seyfert 2 galaxies, this would mark the discovery of yet a new class of high-energy $\gamma$-ray emitters. New data from Fermi/LAT in the coming years will be extremely helpful to search for such emission.

\section{Acknowledgments}

This research has made use of NASA's Astrophysics Data System (ADS), of the SIMBAD database, operated at CDS, Strasbourg, France, and of the NASA/IPAC Extragalactic Database (NED) which is operated by the Jet Propulsion Laboratory, California Institute of Technology, under contract with the National Aeronautics and Space Administration.

\section{References}

[1] A. A. Abdo et al. (Fermi/LAT collaboration), Fermi/Large Area Telescope discovery of gamma-ray emission from a relativistic jet in the narrow-line quasar PMN J0948+0022, ApJ 6999762009 [arXiv:0905.4558]

[2] A. A. Abdo et al. (Fermi/LAT collaboration), Radio-loud narrow-line Seyfert 1 as a new class of gamma-ray active galactic nuclei, ApJ 707 L142 2009 [arXiv: 0911 . 3485]

[3] R. R. J. Antonucci \& J. S. Miller, Spectropolarimetry and the nature of NGC 1068, ApJ 2976211985

[4] D. F. Lester et al., Far-infrared continuum emission from the nucleus, starburst, and extended spiral arms of NGC 1068, ApJ 3217551987

[5] W. Jaffe et al., The central dusty torus in the active nucleus of NGC 1068, Nature 429472004

[6] H. A. Jr. Thronson et al., Near-infrared image of NGC 1068 - Bar-driven star formation and the circumnuclear composition, ApJ 3431581989 
[7] A. A. Abdo et al. (Fermi/LAT collaboration), Fermi Large Area Telescope first source catalog, ApJS 1884052010 [arXiv:1002.2280]

[8] V. Beckmann et al., The second INTEGRAL AGN catalogue, A\&A 5054172009 [arXiv:0907.0654]

[9] W. B. Atwood et al. (Fermi/LAT collaboration), The Large Area Telescope on the Fermi gamma-ray space telescope mission, ApJ 69710712009 [arXiv : 0902 . 1089]

[10] J. R. Mattox et al., The likelihood analysis of EGRET data, ApJ 4613961996

[11] S. D. Barthelmy et al., The burst alert telescope (BAT) on the SWIFT Midex mission, Space Sci. Rev. 1201432005 [astro-ph/0507410]

[12] A. Segreto et al., The Palermo Swift-BAT hard X-ray catalogue. I. Methodology, A\&A 510 A47 2010

[13] A. A. Abdo et al. (Fermi/LAT collaboration), Detection of gamma-ray emission from the starburst galaxies M82 and NGC 253 with the Large Area Telescope on Fermi, ApJ 709 L152 2010 [arXiv:0911.5327]

[14] F. Acero et al. (H.E.S.S. collaboration), Detection of Gamma Rays from a Starburst Galaxy, Science 32610802009 [arXiv: 0909.4651 ]

[15] V. A. Acciari et al. (VERITAS collaboration), A connection between star formation activity and cosmic rays in the starburst galaxy M82, Nature $\mathbf{4 6 2} 7702009$

[16] J.-P. Lenain et al., Seyfert 2 galaxies in the GeV band: jets and starburst, A\&A 524 A72 2010

[17] J. F. Gallimore et al., The parsec-scale radio structure of NGC 1068 and the nature of the nuclear radio source, ApJ 6137942004 [astro-ph / 0406062 ]

[18] J. F. Gallimore et al., A survey of kiloparsec-scale radio outflows in radio-quiet Active Galactic Nuclei, AJ 1325462006 [astro-ph/ 0604219 ]

[19] J.-P. Lenain et al., A synchrotron self-Compton scenario for the very high energy gamma-ray emission of the radiogalaxy M 87. Unifying the TeV emission of blazars and other AGNs ?, A\&A 4781112008 [arXiv:0710.2847]

[20] M. C. Begelman \& M. Sikora, Inverse Compton scattering of ambient radiation by a cold relativistic jet - A source of beamed, polarized continuum in blazars ?, ApJ 3226501987

[21] CTA consortium, Design concepts for the Cherenkov Telescope Array, 2010 [arXiv: 1008 . 3703] 